\title{
Recent Concepts on Cyclic Vomiting Syndrome in Children
}

\author{
Hye Ran Yang, MD
}

Department of Pediatrics, Seoul National University Bundang Hospital, Seoul National University College of Medicine, Seoul, Korea

Cyclic vomiting syndrome (CVS) is a functional gastrointestinal disorder that can occur in both children and adults. Clinical courses of CVS manifesting recurrent severe vomiting episodes and interval illness may affect the long-term quality of life in children with CVS. Therefore, we should be careful in accessing a patient suggestive of CVS. Accurate diagnosis based on diagnostic criteria for CVS and the exclusion from other organic diseases mimicking clinical manifestations of cyclic vomiting is absolutely required. In patients diagnosed as CVS, optimal therapy should be performed to improve symptoms and to reduce complications in prodromal phase and emetic phase, and long-term prophylactic therapy should be tried to prevent the development of vomiting episodes. The identification of triggering factors which induce vomiting episodes might be helpful in preventing vomiting attacks. Systematic approach should be recommended to improve clinical outcome of CVS.

\section{(J Neurogastroenterol Motil 2010;16:139-147)}

\section{Key Words}

Cyclic vomiting syndrome, Diagnosis, Therapy, Child

\section{Introduction}

Cyclic vomiting syndrome (CVS) is a disorder characterized by recurrent episodes of nausea and severe vomiting with symptom-free intervals between the episodes and stereotypic patterns within individuals. ${ }^{1}$ CVS is classified as one of the functional gastrointestinal disorders based on the Rome III. ${ }^{2,3}$ Thorough investigations to distinguish CVS from various organic disorders manifesting with cyclic vomiting, are required.

In this review article, the diagnostic criteria and clinical features of CVS will be investigated, and also recent concepts on the diagnosis, treatment, prophylaxis, and clinical outcomes of CVS will be discussed.

\section{Diagnostic Criteria of \\ Cyclic Vomiting Syndrome}

CVS was first described by $\mathrm{Gee}^{4}$ in the St. Bartholomew's Hospital Reports of 1882. CVS is a functional disorder featured by recurrent discrete episodes of intense nausea and vomiting median 6 times per hour at peak -, lasting hours to days and interval wellness returning to usual status. ${ }^{4}$

The first diagnostic criteria of CVS were set up at the 1 st in-

Received: February 15th, 2010 Accepted: March 26th, 2010

(c) This is an Open Access article distributed under the terms of the Creative Commons Attribution Non-Commercial License (http://creativecommons. org/licenses/by-nc/3.0) which permits unrestricted non-commercial use, distribution, and reproduction in any medium, provided the original work is properly cited.

*Correspondence: Hye Ran Yang, MD

Department of Pediatrics, Seoul National University Bundang Hospital, Seoul National University College of Medicine, 300 Gumi-dong, Bundang-gu, Seongnam, Gyeonggi-do 463-707, Korea

Tel: +82-31-787-7285, Fax: +82-31-787-4054, E-mail: hryang@snubh.org

Financial support: None.

Conflicts of interest: None. 
Table 1. Rome III Criteria for Cyclic Vomiting Syndrome in Children and Adults ${ }^{2,3,7}$

\begin{tabular}{|c|c|}
\hline Children & Adults \\
\hline Must include all of the following: & Must include all of the following: \\
\hline $\begin{array}{l}\text { 1. Two or more periods of intense nausea and unremitting } \\
\text { vomiting or retching lasting hours to days }\end{array}$ & $\begin{array}{l}\text { 1. Stereotypical episodes of vomiting regarding onset (acute) and } \\
\text { duration (less than } 1 \mathrm{wk} \text { ) }\end{array}$ \\
\hline \multirow[t]{5}{*}{ 2. Return to usual state of health lasting weeks to months } & 2. Three or more discrete episodes in the prior year \\
\hline & 3. Absence of nausea and vomiting between episodes \\
\hline & Supportive criterion \\
\hline & History or family history of migraine headaches. \\
\hline & $\begin{array}{l}\text { * Criteria fulfilled for the last } 3 \text { mo with symptom onset at least } 6 \text { mo } \\
\text { before diagnosis }\end{array}$ \\
\hline
\end{tabular}

ternational symposium on CVS held in 1994 . $^{5}$ Thereafter, a revised criteria for CVS at the 2nd international scientific symposium held in 1998, the Rome II criteria in 1999, and the Rome III criteria in 2006 were suggested as new clinical criteria for CVS. $^{2,3,6}$ The most noticeable point is that previous criteria of CVS were only for children, but a newly developed Rome III criteria for functional gastrointestinal disorders is including the diagnostic criteria of CVS for both children and adults (Table $1)^{2,3,7}$

In 2008, new diagnostic criteria for CVS in childhood were suggested as a part of the North American Society for Pediatric Gastroenterology, Hepatology, and Nutrition consensus statement on the diagnosis and management of CVS (Table 2). ${ }^{8}$

\section{Epidemiology of \\ Cyclic Vomiting Syndrome}

CVS had been regarded as a rare disease before, but now it

Table 2. New Diagnostic Criteria for Children With Cyclic Vomiting Syndrome Suggested by the North American Society for Pediatric Gastroenterology, Hepatology, and Nutrition Consensus Statement $^{8}$

All of the criteria must be met to meet this consensus definition of cyclic vomiting syndrome.

1. At least 5 attacks in any interval, or a minimum of 3 attacks during a 6 mo period

2. Episodic attacks of intense nausea and vomiting lasting $1 \mathrm{hr}-10$ days and occurring at least $1 \mathrm{wk}$ apart

3. Stereotypical pattern and symptoms in the individual patient

4. Vomiting during attacks occurs at least 4 times/hr for at least $1 \mathrm{hr}$

5. Return to baseline health between episodes

6. Not attributed to another disorder seems to be relatively common in pediatric population with the prevalence between $1.9 \%$ and $2.3 \%$ according to the data of previous reports. ${ }^{9,10}$ The annual incidence of CVS was 3.15 per 100,000 children for 2005 in Ireland. ${ }^{11}$

There are no data on the prevalence of CVS in adults until now, but recently there are several articles reporting CVS cases in adults. ${ }^{12-14}$ It is assumed that CVS might be more common even in adults than expected before. ${ }^{12}$

CVS has been reported to be slightly more common in female than in male $\left(55: 45\right.$ or 60:40) in all age groups. ${ }^{8,12,15,16}$

CVS can develop in any age, but it has been mainly reported in childhood, with mean age between 4.6 years and 6.9 years. ${ }^{9,16,17}$ There are some reports on adults with CVS, and in adults, mean age at initial diagnosis was 34.8 years. ${ }^{17,18}$ In many patients with CVS, it took several months to years in making a definite diagnosis for CVS.

\section{Clinical Features of \\ Cyclic Vomiting Syndrome}

CVS is clinically characterized by recurrent, stereotypic episodes of intense nausea and vomiting lasting hours to days that are separated by symptom-free intervals lasting weeks to months. ${ }^{4}$ 'Stereotypic' episode means that each episode is similar within individuals as to time of onset, intensity, duration, frequency, and associated symptoms and signs. CVS typically has 3 phases: prodromal phase, emetic phase, and well phase between the episodes. Approaching the patient according to each phase is clinically important to diagnose and treat a patient with CVS.

Approximately $40-80 \%$ of CVS patients are having their own triggering factors evoking vomiting episodes. ${ }^{15,16,19}$ Psychologically excessive stress and emotionally excited state are the most common triggers. Infections (sinusitis, respiratory infection), 
motion sickness, physical exhaustion, excessive exercise, overeating, specific foods (chocolate, cheese, etc.), menstruation, hot weather, and asthmatic attacks also can trigger vomiting episode in CVS patients. ${ }^{16,19}$

About one third of CVS patients show prodromal symptoms before vomiting episodes attack. Prodromal symptoms of CVS consist of change in mood, aura common in migraine, abdominal pain, dyspepsia, nausea, easy fatigability, anxiety, headache, dizziness, etc. ${ }^{9,15,20}$

Vomiting episodes of CVS usually begin at the same time of day stereotypically, especially at dawn and in the morning. ${ }^{9,15,16}$ Vomiting episodes repeat 4-12 times a year showing similar patterns in each patient. ${ }^{9,19,21}$ Episodes usually continue from 2 hours to 10 days, and CVS patients show intensive emesis that develop minimum 4 vomiting per hour during the first hours of each episode.

Every vomiting episode eventually resolves spontaneously even if left untreated. Most patients return to usual health condition as soon as the episode stops. However, more than one third of CVS patients can show vomiting symptoms even during well phase between the vomiting episodes.

Intense vomiting during the episodes is associated with other symptoms in most patients with CVS. Nausea and abdominal pain on periumbilical or epigastric area are the most common associated symptoms. Headache, mild fever, loose stools, excessive drooling, photophobia, intolerance to noise, pallor, tachycardia,

Table 3. Comparison of Clinical Features Between Children and Adults With Cyclic Vomiting Syndrome (Adapted and Modified From Abell et al. ${ }^{17}$ )

\begin{tabular}{llc}
\hline & Children & Adults \\
\hline Age at diagnosis & $4.6-5.3 \mathrm{yr}$ & $34.8 \mathrm{yr}$ \\
Delay in diagnosis & $2.6-3.1 \mathrm{yr}$ & $7.9 \mathrm{yr}$ \\
$\begin{array}{l}\text { Duration of episodes } \\
\text { Interepisode intervals }\end{array}$ & 2.0 days & 3.8 days \\
Prodromal symptoms & Common & Common \\
Symptoms during episode & & \\
Vomiting & Universal & Universal \\
Abdominal pain & Common & Common \\
Interepisodic nausea & Rare & Common \\
or dyspepsia & & \\
Associated psychiatric & Common & Common \\
manifestations & & \\
Migraine headache & Common & Common \\
or family history of & & \\
migraine headache & & \\
\hline
\end{tabular}

and mild elevation of blood pressure are also common. ${ }^{16,18,21}$ Leukocytosis is often observed during vomiting episodes in CVS patients.

Various complications may occur related to vomiting symptoms. Massive prolonged vomiting can cause dehydration and electrolyte imbalance in emetic phase. Gastritis, esophagitis, Mallory-Weiss tear, weight loss, dental caries, and psychiatric problems including social withdrawal and depression, can be complicated secondary to intensive vomiting episodes. ${ }^{20,22}$

Clinical manifestations of CVS in children are somewhat different from those of adult patients (Table 3). ${ }^{17}$ Duration of each vomiting episodes are shorter in children than in adults. ${ }^{12,17}$ Interepisodic nausea or dyspepsia is rare in children with CVS, but common in adult patients. ${ }^{17}$ Some adult patients are initially misdiagnosed as gastroparesis due to clinical similarity. Namin et al. ${ }^{14}$ reported that $5 \%$ of adult patients suspected to be gastroparesis finally turn out to be CVS. Therefore, the diagnosis for CVS is more frequently delayed in adults than in children.

However, in a study by Prakash et al. in $2001{ }^{13}$ it was regarded that there were striking similarities in clinical manifestations across age groups. The only difference was the duration of vomiting episodes that lengthened progressively from infancy into adulthood. ${ }^{13}$

\section{Associated Disorders with Cyclic Vomiting Syndrome}

Migraine and motion sickness are commonly associated with vomiting episodes of CVS. According to previous studies, $11-21 \%$ of CVS patients accompany migraine headache, and 29-46\% accompany motion sickness. ${ }^{9,20}$ Migraine and family history of migraine were noted in $39-81 \%$ of CVS patients in childhood, and in $24-70 \%$ of adult patients. ${ }^{17}$

Even more, both irritable bowel syndrome and family history of irritable bowel syndrome are more common in CVS patients (67\% vs. $62 \%)$ than in general population (10-20\% vs. $14 \%){ }^{23}$

Epilepsy is approximately 10 times more common in CVS patient than in general population. ${ }^{16,23}$ However, there was no CVS patient showing epileptic seizure in Korea. ${ }^{15}$

Children with CVS often accompany psychiatric disorders such as anxiety disorders or depression. ${ }^{24}$

Family history of CVS was also common in CVS patients (3\%) than in general population $(0.4-2.0 \%){ }^{23}$ 


\section{Differential Diagnosis of Cyclic Vomiting Syndrome}

Cyclic vomiting occurs not only in CVS patients but also in other organic diseases clinically mimicking CVS. Therefore, the diagnosis of CVS should be done very carefully, ruling out all possible organic causes of cyclic vomiting. Previous study reported that among patients manifested as cyclic vomiting, $5 \%$ had gastrointestinal disorders, $7 \%$ were due to other organic disorders, and only $88 \%$ were compatible with CVS. ${ }^{25}$

Clinically differential diagnosis of CVS includes gastrointestinal disorders such as bowel obstruction, inflammatory disease of gastrointestinal tracts, pancreatic diseases, and hepatobiliary diseases; infectious diseases such as parasite infestation and protozoa infection, otitis media, chronic sinusitis, and hepatitis; neurologic disorders such as migraine, epilepsy, and space occupying CNS lesions; metabolic and endocrine disorders such as diabetes mellitus, Addison disease, pheochromocytoma, aminoaciduria, organic aciduria, fatty acid oxidation disorders, mi- tochondrial disorders, and urea cycle defects; medications and toxins such as antibiotics, NSAIDs, laxatives, high dose fat soluble vitamins, hormones, etc.; urologic/gynecologic disorders such as pelvi-ureteric junction obstruction and nephrolithiasis; and miscellaneous disorders and psychiatric disorders (Table 4). ${ }^{26}$

\section{Suggested Hypotheses on the Pathogenesis of Cyclic Vomiting Syndrome}

The etiology and pathogenesis of CVS still remain unknown. It is assumed that various factors can affect the development of vomiting episodes in CVS, and severe hypotheses have been suggested as possible etiologies: migraine variant, ${ }^{27}$ mitochondrial diseases including mitochondrial fatty acid oxidation disorders, ${ }^{28,29}$ gastrointestinal motility disorder, ${ }^{30}$ corticotropin-releasing factor in response to stress, ${ }^{31}$ disorder of the brain-gut axis, ${ }^{32}$ autonomic dysfunction, ${ }^{33}$ abdominal epilepsy, ion channel dysfunction, and altered psychodynamics, etc.

Recent concept on CVS is mitochondrial energy depletion due to mitochondrial mutation along with precipitating stress or

Table 4. Differential Diagnosis of Cyclic Vomiting in Children and Adolescents (Adapted and Modified From Forbes et al. ${ }^{26}$ )

Gastrointestinal disorders

Bowel obstruction (malrotation with volvulus, internal hernia, duplication cyst, and intermittent duodenal intussuception)

Inflammatory diseases (gastritis, duodenitis, peptic ulcer disease, chronic appendicitis, and inflammatory bowel disease)

Pancreatid diseases (pancreatitis and pancreatic pseudocyst)

Hepatobiliary disease (hepatitis)

Infections

Enteric agents (Giardiasis, Gastrospirillium, Entamoeba coli, Blastocystis hominis, and pinworms)

Otitis media, chronic sinusitis, and hepatitis

Neurologic disorders

Migraine

Epilepsy

Space occupying central nervous system lesions (hydrocephalus, posterior fossa tumors, subdural hematoma, and subdural effusion)

Familial dysautonomia

Metabolic and endocrine disorders

Diabetes mellitus, Addison disease, and pheochromocytoma

Aminoaciduria, organic aciduria, fatty acid oxidation disorders, mitochondrial disorders, and urea cycle defects

Medications and toxins

Antibiotics, non-steroidal anti-inflammatory drugs, laxatives, high dose fat soluble vitamins, hormones, etc.

Urologic/gynecologic disorders

Pelvi-ureteric junction obstruction

Nephrolithiasis

Miscellaneous disorders

Abdominal migraines

Abdominal epilepsy

Hypothalamic surge

Asthma

Benign paroxysmal positional vertigo 
excitement may predispose the onset of vomiting episodes in patients with $\mathrm{CVS}^{34}$

Genetic association including A3243G mitochondrial DNA mutation was also suggested as the possible cause of $\mathrm{CVS}^{35}$

Food allergy was also suggested as a possible pathogenesis of CVS by Lucarelli et al. ${ }^{36}$ in 2000 . However, there have been no more studies on the relationship between CVS and food allergy; it is yet controversial.

\section{Diagnostic Evaluation for \\ Children with Cyclic Vomiting}

Thorough history taking and physical examination are essential to diagnose the CVS and to rule out organic diseases causing cyclic vomiting. Alarm symptoms and signs are important clues for the differential diagnosis of cyclic vomiting in children. Suspicious alarm symptoms and signs clinically suggestive of organic diseases are bilious vomiting, severe abdominal pain accompanied by abdominal tenderness, hematemesis, attacks precipitated by acute illness and prior fasting or high protein meal, neurologic symptoms and signs (such as irritability, altered mental status, seizure, abnormal eye movements, papilledema, and motor asymmetry), and progressively worsening vomiting episodes. ${ }^{8}$ These symptoms and signs are mainly indicating the possibility of significant gastrointestinal diseases, metabolic disorders, neurologic diseases, or other organic diseases manifesting cyclic vomiting. ${ }^{8}$

Laboratory studies including complete blood count, liver function tests, electrolytes, glucose, blood urea nitrogen, creatinine, amylase, lipase, ammonia, urinalysis, and pregnancy test for adolescents, are basically recommended in all patients.

Laboratory metabolic findings include hypoglycemia, substantial anion gap metabolic acidosis, respiratory alkalosis, or hyperammonemia especially during vomiting episodes. ${ }^{8}$ Therefore, if clinically indicated, additional laboratory tests such as lactate, pyruvate, organic acid and amino acid analysis, plasma carnitine and acylcarnitine, plasma cortisol levels, and urinary prophyrins can be performed to exclude the possibility of metabolic and endocrine disorders. ${ }^{8}$

Barium contrast study of upper gastrointestinal tract with small bowel follow through, abdominal sonography, or abdominal computed tomography are useful radiologic methods to find out organic lesions possibly which can cause cyclic vomiting features. Barium studies are usually performed to rule out organ-
History taking and physical examination

- Diagnostic criteria of cyclic vomiting syndrome in suspicious
children manifesting with cyclic vomiting
- Alam symptoms and signs indicating organic diseases

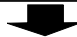

Initial laboratory tests and radiologic studies

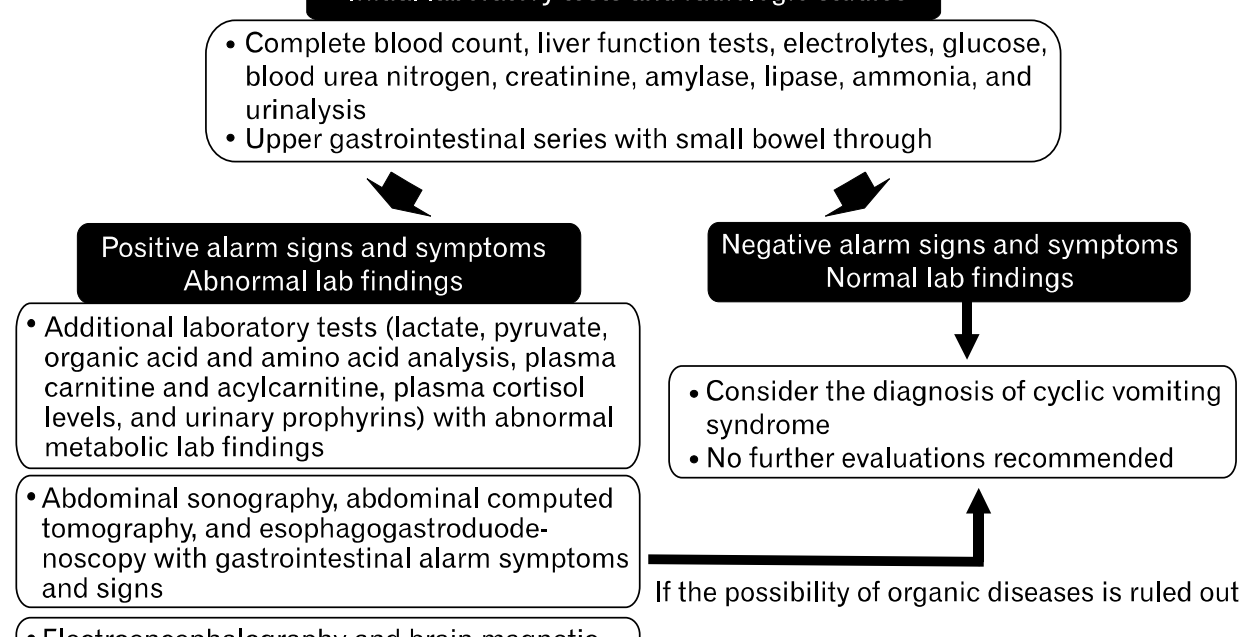

- Electroencephalography and brain magnetic resonance imaging with abnormal neurologic findings on history and neurologic examination
Figure 1. Recommended diagnostic algorithm of cyclic vomiting syndrome in children. 
ic gastrointestinal diseases including chronic intestinal pseudo-obstruction and malrotation. ${ }^{17}$ Esophagogastroduodenoscopy is recommended to rule out upper gastrointestinal disorders like peptic ulcer diseases.

If a patient reveals neurologic manifestations, electroencephalography and brain magnetic resonance imaging can be excellent diagnostic tools to detect intracranial abnormalities. ${ }^{8}$

Recommended diagnostic algorithm of the CVS in children is shown in Figure 1.

\section{Strategies for the Treatment of Cyclic Vomiting Syndrome}

\section{Prophylactic therapy in prodromal phase}

When children diagnosed as CVS begin to manifest prodromal symptoms, various therapeutic trials can be applied to prevent the attack of vomiting episode. These kinds of prophylactic therapy in prodromal phase includes the control of stressful life style (lying down in a dark, quiet environment or hot bath), high carbohydrate ingestion, anti-emetic agents (ondansetron and granisetron), anti-anxiety agents (lorazepam), anti-migraine agents (sumatriptan and zolmitriptan), ${ }^{17}$ and has been mainly tried in pediatric patients. However, the effectiveness of these therapies was not proved due to the lack of data.

Recommended medications during prodromal phase of CVS are as follows: $5-\mathrm{HT}_{3}$ receptor antagonists (ondansetron and granisetron), anti-histamines (diphenhydramine), phenothiazines (promethazine), benzodiazepines (lorazepam), and anti-migraine agent (sumatriptan). ${ }^{8,17}$

Recently, the Pediatric Guidelines Committee of North American Society for Pediatric Gastroenterology, Hepatology and Nutrition commented on the efficacy of anti-migraine $5 \mathrm{HT}_{1 \mathrm{~B} / \mathrm{ID}}$ receptor agonists (sumatriptan) during prodromal phase or early phase of emetic episodes in children with family history of migraine. ${ }^{8}$ Actually, this $5 \mathrm{HT}_{1 \mathrm{~B} / 1 \mathrm{D}}$ receptor agonist was not approved for use in children and adolescents younger than 18 years of age. However, it is recommended to try out this agent as early as possible to terminate an episode in children 12 years and older who have mild episodes less than 1 episode per month. ${ }^{8,37}$

\section{Medical treatment for cyclic vomiting syndrome in emetic phase}

No treatment has been proved to be effective in controlling emetic phase of CVS until now. The goal of therapy during emetic phase is the termination of vomiting episodes as soon as possible for a patient to return to usual life and the prevention of complications such as dehydration and electrolyte imbalance, caused by intensive vomiting.

In acute phase of emetic episodes, patients should be admitted to the hospital to provide dark, quiet surroundings and to prevent severe dehydration and correct electrolyte imbalance with hydration of intravenous fluids. Gastric acid suppressing agents including proton pump inhibitors (omeprazole) and $\mathrm{H}_{2}$ receptor antagonists (ranitidine) may be helpful in preventing mucosal damage of the esophagus.

Intravenous anxiolytic agent, lorazepam (0.05 to 0.1 $\mathrm{mg} / \mathrm{kg} /$ dose every $6 \mathrm{hr}$ ) is effective in suppressing vomiting symptoms or sedate patients in acute emetic episodes. Intravenous injection of $5-\mathrm{HT}_{3}$ receptor antagonists ondansetron ( 0.3 to $0.4 \mathrm{mg} / \mathrm{kg} / \mathrm{dose}$ every 4 to $6 \mathrm{hr}$, upper limit of $20 \mathrm{mg} / \mathrm{dose}$ ) and granisetron, or anti-histamine diphenhydramine (1.0 to $1.25 \mathrm{mg}$ / $\mathrm{kg} /$ dose every $6 \mathrm{hr}$ ) can be tried to reduce vomiting symptoms. ${ }^{8,17}$

Table 5. Recommended Prophylactic Drugs for Children With Cyclic Vomiting Syndrome (Adapted and Modified From NASPGHAN consensus ${ }^{8}$ )

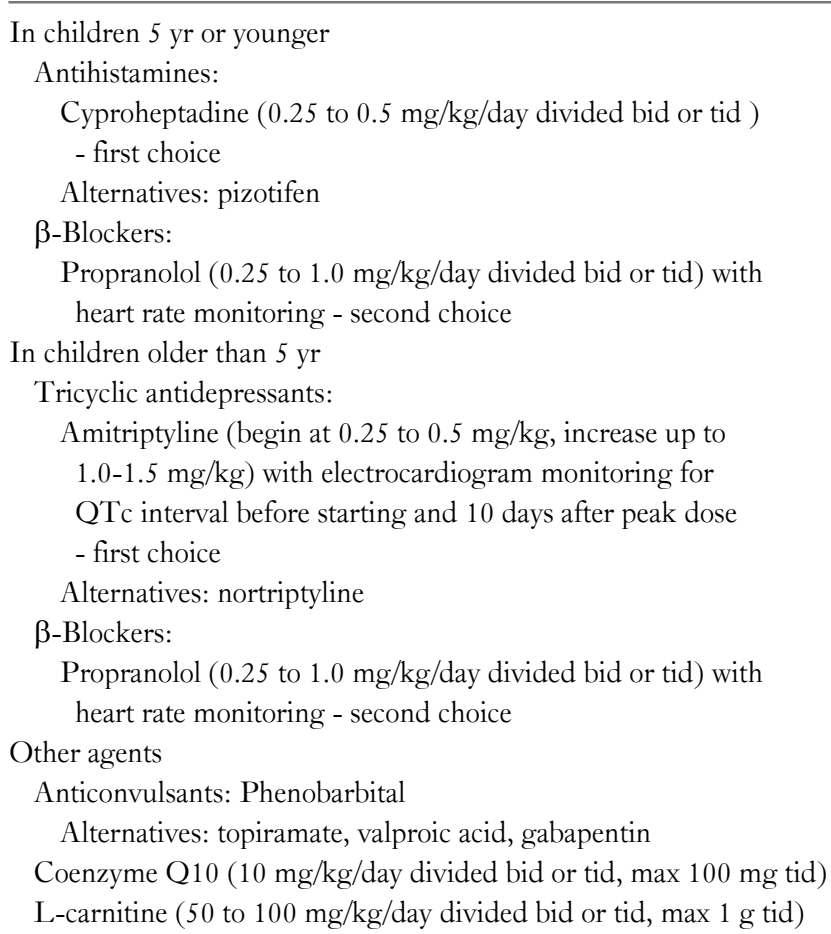

NASPGHAN, North American Society for Pediatric Gastroenterology, Hepatology, and Nutrition; QTc, corrected QT. 


\section{Prophylactic therapy during well phase}

The goal of long-term prophylactic therapy of CVS is to prevent repetitive vomiting episodes or to decrease the severity of symptoms during episodes, thus, allowing patients to continue normal life.

Prophylaxis of symptomatic vomiting episodes is focused on the identification and avoidance of triggering factors and prophylactic drugs. If precipitants causing vomiting episodes can be identified in each patient, it may be possible to prevent the attacks of episodes by avoiding stressful events, infection, specific foods, sleep deprivation, triggering foods (chocolate and cheese), and motion sickness. ${ }^{26}$ Life style modification also include avoidance of energy-depleted states (fasting, acute infection, and acute illness) based on Boles' hypothesis. ${ }^{35}$

Medical prophylaxis in children with CVS is composed of various medicines that are known to be somewhat effective in preventing vomiting episodes: amitriptyline, cyproheptadine, propranolol, etc. (Table 5). ${ }^{8,17}$

Based on the similarity in clinical features between CVS and migraine, anti-migraine agents (cyproheptidine, pizotifen, propranolol, etc.) can be tried especially in patients with migraine headache or family history of migraine. ${ }^{38}$ Cyproheptadine, anti-histamine and serotonin receptor antagonist is effective in young children and is the first choice in children 5 years old or less. ${ }^{8,39}$ And, $\beta$-blocker propranolol is recommended as the second choice prophylactic drug in children with CVS of all ages. ${ }^{8}$

Tricyclic anti-depressive agent, amitriptyline is more effective as prophylactic drugs in children older than 5 years and adults. $^{39,40}$

Although most prophylactic medications are relatively safe even in children, heart rate monitoring and electrocardiogram monitoring are required while administering and dosing up propronolol and amitriptyline because these drugs can cause bradycardia and arrhythmia, respectively, as side effects. ${ }^{8}$

There were several trials on other prophylactic agents. In a study by Vanderhoof et al., ${ }^{41}$ oral erythromycin $(20 \mathrm{mg} / \mathrm{kg} / \mathrm{day})$ was tried to patients with CVS as a prokinetic agent, which was suggested to be effective in some patients, but the efficacy was not confirmed due to poor quality of the study.

Anti-epileptic drugs were not effective regardless of electroencephalogram finding, however, a few studies are reporting the efficacy of valproic acid ${ }^{42}$ and phenobarbital. ${ }^{43}$

Recently, alternative prophylactic medications including coenzyme Q10 and carnitine are suggested to be possibly effective. $^{44,45}$

It is difficult to predict the response to medication in individuals. Therefore, various factors that can affect the efficacy of therapy, that is, age, co-morbid diseases, drug dosage, and adverse effects of drugs, should be considered before initiating prophylactic medication. ${ }^{8}$ When the diagnosis for CVS is confirmed based on the diagnostic criteria and diagnostic testing, individualized therapy can be tried with a first-line drug, starting from low dose and carefully increasing every 1 to 4 weeks up to therapeutic range, for at least 2 cycles of vomiting episodes. ${ }^{8}$ If the medication is not effective in preventing the attack of episodes or if there are significant adverse effects, the second-line drug or combination therapy should be considered. However, long-term prophylactic strategies are not confirmed yet.

Further studies are needed in the future on the effective prophylaxis of CVS based on its pathogenesis.

\section{Long-term Outcome of Cyclic Vomiting Syndrome in Children}

It is not easy to predict clinical course and long-term outcome of CVS in children due to lack of data. Many patients eventually recover from CVS, but some patients suffer from migraine headache later. And, vomiting episodes are repeated even in adulthood in more than one third of CVS children. ${ }^{15,46,47}$ Therefore, regular follow-up and monitoring of disease courses are required in children with CVS for a long time or maybe lifelong in some patients.

\section{Conclusion}

Because CVS is a relatively common functional gastrointestinal disorder in children, we should be careful in approaching the patients manifesting cyclic vomiting. CVS has its own unique clinical features of recurrent severe vomiting episodes and intervening wellness, which can affect long-term morbidity in patients. Early diagnosis applying the diagnostic criteria for CVS to rule out organic diseases is important in patients. Recommended therapeutic strategies for CVS are targeting the reduction of symptoms and complications in prodromal phase and emetic phase. Long-term prophylaxis is focused on the prevention of vomiting episodes. Individualized systematic approach is recommended in children with CVS for accurate diagnosis and better clinical outcome. 


\section{References}

1. Rasquin-Weber A, Hyman PE, Cucchiara S, et al. Childhood functional gastrointestinal disorders. Gut 1999;45(suppl 2):1160-1168.

2. Hyman PE, Milla PJ, Benninga MA, Davidson GP, Fleisher DF, Taminiau J. Childhood functional gastrointestinal disorders: neonate/toddler. Gastroenterology 2006;130:1519-1526.

3. Rasquin A, Di Lorenzo C, Forbes D, et al. Childhood functional gastrointestinal disorders: child/adolescent. Gastroenterology 2006; 130:1527-1537.

4. Gee S. On fitful or recurrent vomiting. St Bartholomew's Hospital Rep 1882;18:1-6.

5. Diagnostic criteria for cyclic vomiting syndrome. Proceedings of the International Scientific Symposium on Cyclic Vomiting Syndrome. J Pediatr Gastroenterol Nutr 1995;21(suppl 1):vi.

6. Drossman DA. The functional gastrointestinal disorders and the Rome III process. Gastroenterology 2006;130:1377-1390.

7. Tack J, Talley NJ, Camilleri M, et al. Functional gastroduodenal disorders. Gastroenterology 2006;130:1466-1479.

8. Li BU, Lefevre F, Chelimsky GG, et al. North American Society for Pediatric Gastroenterology, Hepatology, and Nutrition consensus statement on the diagnosis and management of cyclic vomiting syndrome. J Pediatr Gastroenterol Nutr 2008;47:379-393.

9. Abu-Arefeh I, Russell G. Cyclic vomiting syndrome in children: a population-based study. J Pediatr Gastroenterol Nutr 1995;21:454458.

10. Ertekin V, Selimoğlu MA, Altnkaynak S. Prevalence of cyclic vomiting syndrome in a sample of Turkish school children in an urban area. J Clin Gastroenterol 2006;40:896-898.

11. Fitzpatrick E, Bourke B, Drumm B, Rowland M. The incidence of cyclic vomiting syndrome in children: population-based study. Am J Gastroenterol 2008;103:991-995.

12. Fleisher DR, Gornowicz B, Adams K, Burch R, Feldman EJ. Cyclic vomiting syndrome in 41 adults: the illness, the patients, and problems of management. BMC Med 2005;3:20.

13. Prakash C, Staiano A, Rothbaum RJ, Clouse RE. Similarities in cyclic vomiting syndrome across age groups. Am J Gastroenterol 2001;96:684-688.

14. Namin F, Patel J, Lin Z, et al. Clinical, psychiatric and manometric profile of cyclic vomiting syndrome in adults and response to tricyclic therapy. Neurogastroenterol Motil 2007;19:196-202.

15. Yang HR, Ko JS, Seo JK. A Study on the disease course and prognosis of cyclic vomiting syndrome. J Korean Pediatr Soc 2001;44: 1141-1156.

16. Fleisher DR, Matar M. The cyclic vomiting syndrome: a report of 71 cases and literature review. J Pediat Gastroenterol Nutr 1993; 17:361-369.

17. Abell TL, Adams KA, Boles RG, et al. Cyclic vomiting syndrome in adults. Neurogastroenterol Motil 2008;20:269-284.

18. Prakash C, Staiano A, Rothbaum RJ, Clouse RE. Similarities in cyclic vomiting syndrome across age groups. Am J Gastroenterol 2001; 96:684-688.

19. Fleisher DR. Cyclic vomiting syndrome: a paroxismal disorder of brain-gut interaction. J Pediatr Gastroenterol Nutr 1997;25(suppl
1):S13-S15

20. Fleisher DR. The cyclic vomiting syndrome described. J Pediatr Gastroenterol Nutr 1995;21(suppl 1):S1-S5.

21. Li BU, Fleisher DR. Cyclic vomiting syndrome: features to be explained by a pathophysiologic model. Dig Dis Sci 1999;44(suppl): 13S-18S.

22. Forbes D. Differential diagnosis of cyclic vomiting syndrome. J Pediatr Gastroenterol Nutr 1995;21(suppl 1):S11-S14.

23. Pareek N, Fleisher DR, Abell T. Cyclic vomiting syndrome: what a gastroenterologist needs to know. Am J Gastroenterol 2007;102: 2832-2840.

24. Jones MP, Crowell MD, Olden KW, Creed F. Functional gastrointestinal disorders: an update for the psychiatrist. Psychosomatics 2007;48:93-102.

25. Li BU, Murray RD, Heitlinger LA, Robbins JL, Hayes JR. Heterogeneity of diagnoses presenting as cyclic vomiting. Pediatrics 1998;102:583-587.

26. Forbes D, Fairbrother S. Cyclic nausea and vomiting in childhood. Aust Fam Physician 2008;37:33-36.

27. Welch KM. Scientific basis of migraine: speculation on the relationship to cyclic vomiting. Dig Dis Sci 1999;44(suppl):26S-30S.

28. Boles RG, Williams JC. Mitochondrial disease and cyclic vomiting syndrome. Dig Dis Sci 1999;44(suppl):103S-107S.

29. Rinaldo P. Mitochondrial fatty acid oxidation disorders and cyclic vomiting syndrome. Dig Dis Sci 1999;44(suppl):97S-102S.

30. Beck NS. Abnormal electrogastrography and gastric emptying time in patients with cyclic vomiting syndrome during symptom free interval. J Korean Pediatr Soc 1997;40:209-216.

31. Taché Y. Cyclic vomiting syndrome: the corticotropin-releasing-factor hypothesis. Dig Dis Sci 1999;44(suppl):79S-86S.

32. Li BU, Misiewicz L. Cyclic vomiting syndrome: a brain-gut disorder. Gastroenterol Clin North Am 2003;32:997-1019.

33. Rashed H, Abell TL, Familoni BO, Cardoso S. Autonomic function in cyclic vomiting syndrome and classic migraine. Dig Dis Sci 1999;44(supple):74S-78S.

34. Boles RG, Adams K, Li BU. Maternal inheritance in cyclic vomiting syndrome. Am J Med Genet A 2005;133:71-77.

35. Salpietro CD, Briuglia S, Merlino MV, Di Bella C, Rigoli L. A mitochondrial DNA mutation (A3243G mtDNA) in a family with cyclic vomiting. Am J Pediatr 2003;162:727-728.

36. Lucarelli S, Corrado G, Pelliccia A, et al. Cyclic vomiting syndrome and food allergy/intolerance in seven children: a possible association. Eur J Pediatr 2000;159:360-363.

37. Lewis D, Ashwal S, Hershey A, Hirtz D, Yonker M, Silberstein S. Practice parameter: pharmacological treatment of migraine headache in children and adolescents: report of the American Academy of Neurology Quality Standards Subcommittee and the Practice Committee of the Child Neurology Society. Neurology 2004;63: 2215-2224.

38. Withers GD, Silburn SR, Forbes DA. Precipitants and aetiology of cyclic vomiting syndrome. Acta Paediatr 1998;87:272-277.

39. Andersen JM, Sugerman KS, Lockhart JR, Weinberg WA. Effective prophylactic therapy for cyclic vomiting syndrome in children using amitriptyline or cyproheptadine. Pediatrics 1997;100: 977-981.

40. Prakash C, Clouse RE. Cyclic vomiting syndrome in adults: clinical 
features and response to tricyclic antidepressants. Am J Gastroenterol 1999;94:2855-2860.

41. Vanderhoof JA, Young R, Kaufman SS, Ernst L. Treatment of cyclic vomiting in childhood with erythromycin. J Pediatr Gastroenterol Nutr 1995;21:(suppl 1):S60-S62.

42. Hikita T, Kodama H, Nakamoto N, et al. Effective prophylactic therapy for cyclic vomiting syndrome in children using valproate. Brain Dev 2009;31:411-413.

43. Gokhale R, Huttenlocher PR, Brady L, Kirschner BS. Use of barbituates in the treatment of cyclic vomiting during childhood. J Pediatr Gastroenterol Nutr 1997;25:64-67.
44. Boles RG, Lovett-Barr MR, Preston A, Li BU, Adams K. Treatment of cyclic vomiting syndrome with co-enzyme Q10 and amitriptyline, a retrospective study. BMC Neurol 2010;10:10.

45. Van Calcar SC, Harding CO, Wolff JA. L-carnitine administration reduces number of episodes in cyclic vomiting syndrome. Clin Pediatr (Phila) 2002;41:171-174.

46. Hammond J. The late sequale of recurrent vomiting of childhood. Dev Med Child Neurol 1974;16:15-22.

47. Scobie BA. Recurrent vomiting in adults, a syndrome? Med J Aust 1983;1:329-331. 